\title{
Sliding Mode Control of an Isolated Wound Rotor Synchronous Generator
}

\author{
A. Dòria-Cerezo, E. Fossas, R.S. Muñoz-Aguilar and V.I. Utkin
}

\begin{abstract}
This paper presents a sliding mode controller for a wound rotor synchronous machine, acting as a generator for an isolated load. The standard dq-model of the machine is connected to a resistive load. A sliding surface is defined in order to fulfill control objectives, and the Ideal Sliding Dynamics is proved to be stable. From the desired surface, the standard sliding methodology is applied, and a robust controller is obtained. Numerical simulations are also presented to validate the control law.
\end{abstract}

\section{INTRODUCTION}

The wound rotor synchronous machine (WRSM) is used for generation and also for driving applications [1]. In the generation case the field voltage is used for regulating the stator voltage, while in motor applications this variable can be used to control the reactive power of the machine [2]. Several techniques are proposed for controlling the WRSM. Linear techniques are the most used in the industry [3][4], but decoupling methods [5], widely employed for asynchronous machines, have also been extended to the synchronous case. Recently, modern non-linear techniques have been used for controlling this machine, as passivity-based techniques [6], or Sliding Mode Control [7].

In general, sliding mode technique is appropriate for variable structure systems (VSS) as power converters [8]. Even that electrical machines are not VSS, Sliding Mode Control has been proposed as an appropriate technique for controlling them [9]. Mainly, this is because of the use of power converters in applying the electrical machines control voltages and to the discrete values the voltage take.

There are several sliding mode controllers reported in the literature for synchronous machines operating both in motor and generator modes (motor drive applications [10], wind energy systems [11]). The special case of the WRSM is not widely studied. In [7] a WRSM (with damping windings) is considered as a generator directly connected to the grid.

The main goal of this work is to design a sliding mode control algorithm for a wound rotor synchronous generator

A. Dòria-Cerezo is with the Department of Electrical Engineering and the Institute of Industrial and Control Engineering, Universitat Politècnica de Catalunya, 08800 Vilanova i la Geltrú, Spain arnau.doria@upc.edu

E. Fossas is with the Institute of Industrial and Control Engineering, Universitat Politècnica de Catalunya, 08028 Barcelona, Spain enric.fossas@upc.edu

R.S. Muñoz-Aguilar is with the Department of Electrical Engineering and the Institute of Industrial and Control Engineering, Universitat Politècnica de Catalunya, 08222 Terrassa, Spain raul.munoz-aguilar@upc.edu

V.I. Utkin is with the Department of Electrical Engineering, The Ohio State University, OH 43210 USA utkin@ee.eng.ohio-state.edu for an isolated load. Usually generation with WRSM is studied for a grid connection, see for example [7]. In this case, the stator voltage and frequency are fixed by the power grid, while the rotor voltage helps to improve the power factor that is, to compensate the reactive power at the connection point. The stand-alone case is essentially different: neither amplitude nor frequency of stator voltage are externally fixed. While the mechanical speed determines the frequency, the rotor voltage must fix the stator amplitude. As far to our knowledge, control designs for this kind of configuration are not reported in the literature. Usually, industry applications use linear controllers which cannot ensure stability. In this paper it is shown that the classic sliding mode control technique is enough to ensure stability and also provides a fast response. As a first approximation, a resistive load is considered here in order to consider much complex loads (non-linear and dynamic loads) in future works.

The paper is organized as follows. In Section II the wound rotor synchronous machine model is introduced and its control goals are described. In Section III, the equilibrium points are computed, the Ideal Sliding Dynamics is analyzed and the Sliding Mode controller is obtained. The simulation results are included in Section IV and, finally, conclusions are stated in Section V.

\section{SYSTEM DESCRIPTION}

In this Section the dynamic model of the system is presented. Figure 1 shows the proposed scenario: an Internal Combustion Engine (ICE) drags a WRSM, which acts as a generator to fed an isolated load, where $\omega$ is the mechanical speed, $v_{s}, v_{F}$ are the stator and field voltages, and $i_{s}, i_{F}$ are the stator and field currents.

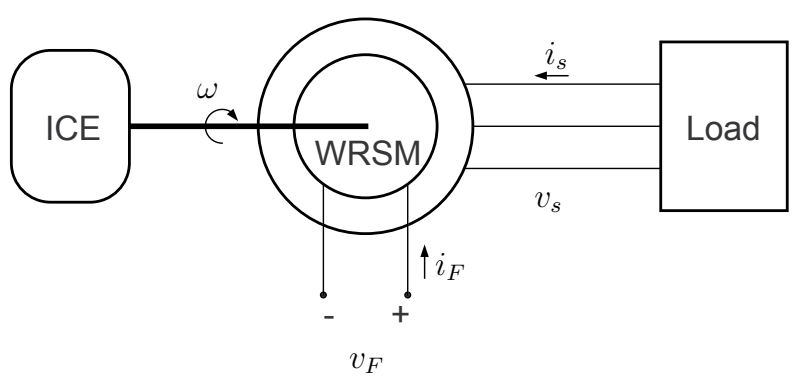

Fig. 1. Scheme of wound rotor synchronous generator with an isolated load.

As explained before, this system differs from the typical grid connection in that, in the last case, the frequency and the voltage amplitude are fixed by the grid. For an isolated connection the frequency is determined by the mechanical 
speed (provided by the ICE), while the voltage amplitude must be assured by the rotor field voltage.

\section{A. Dynamic model}

From the well-known dynamical equations (in the dq coordinates) of the WRSM, and the interconnection rules with a pure resistor load, the whole dynamical system is presented.

Let us to use a dq reference given by the mechanical speed. Then, assuming that the mechanical speed is externally regulated by the ICE, the electrical part of the wound rotor synchronous machine can described as

$$
L \frac{\mathrm{d} x}{\mathrm{~d} t}=\left(\begin{array}{ccc}
-R_{s} & \omega L_{s} & 0 \\
-\omega L_{s} & -R_{s} & -\omega L_{m} \\
0 & 0 & -R_{F}
\end{array}\right) x+\left(\begin{array}{c}
v_{d} \\
v_{q} \\
v_{F}
\end{array}\right)
$$

where

$$
L=\left(\begin{array}{ccc}
L_{s} & 0 & L_{m} \\
0 & L_{s} & 0 \\
L_{m} & 0 & L_{F}
\end{array}\right)
$$

is the inductance matrix, $x^{T}=\left(i_{d}, i_{q}, i_{F}\right) \in \mathbb{R}^{3}$ are the dqstator and field currents, $R_{s}$ and $R_{F}$ are the stator and field resistances, $L_{s}, L_{m}$ and $L_{F}$ are the stator, mutual and field inductances, $\omega$ is the mechanical speed ${ }^{1}, v_{d}, v_{q}$ and $v_{F}$ are the dq-stator voltages and the field voltage which will be used as a control input.

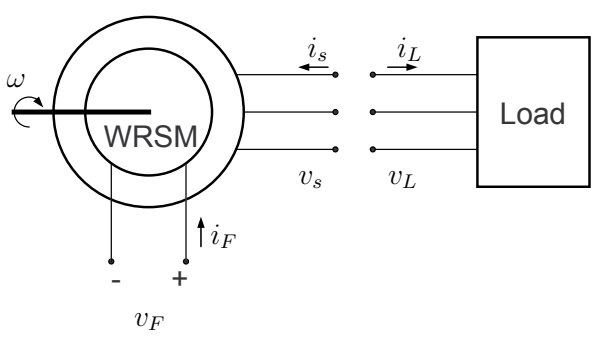

Fig. 2. Detail of the interconnection between a wound rotor synchronous machine and a load.

In order to design the control law, let us obtain the complete model of a WRSM connected to a resistive load $R_{L}$. The interconnection scheme is depicted in Figure 2, where $v_{L}^{T}=\left(v_{L d}, v_{L q}\right) \in \mathbb{R}^{2}$ and $i_{L}^{T}=\left(i_{L d}, i_{L q}\right) \in \mathbb{R}^{2}$ are the dq load voltages and currents, which are related by

$$
\left(\begin{array}{c}
v_{L d} \\
v_{L q}
\end{array}\right)=R_{L}\left(\begin{array}{c}
i_{L d} \\
i_{L q}
\end{array}\right)
$$

where $R_{L}$ is the resistance value. The interconnection rules, according to Fig. 2 are

$$
\begin{aligned}
& v_{s}=v_{L} \\
& i_{L}=-i_{s} .
\end{aligned}
$$

Now, putting together (1) and (2), the system can be written in an affine form as

$$
L \frac{\mathrm{d} x}{\mathrm{~d} t}=A x+G u,
$$

\footnotetext{
${ }^{1}$ For simplicity it is considered a 2-poles machine.
}

where $u=v_{F}, L$ is the inductance matrix defined before,

$$
A=\left(\begin{array}{ccc}
-\left(R_{s}+R_{L}\right) & \omega L_{s} & 0 \\
-\omega L_{s} & -\left(R_{s}+R_{L}\right) & -\omega L_{m} \\
0 & 0 & -R_{F}
\end{array}\right),
$$

and

$$
G=\left(\begin{array}{l}
0 \\
0 \\
1
\end{array}\right)
$$

\section{B. Control objective}

As mentioned before, this machine must ensure stator voltage amplitude and frequency. For a synchronous machine, the stator frequency is directly given by the mechanical speed, which, in this paper is assumed to be constant and externally regulated. Then, the system output is the stator voltage amplitude $V_{s}$, which can be easily obtained, in a dq-framework as

$$
V_{s}=\sqrt{v_{d}^{2}+v_{q}^{2}} .
$$

Last equation can be expressed in current terms using (2), which yields

$$
V_{s}=R_{L} \sqrt{i_{d}^{2}+i_{q}^{2}},
$$

and the control input is the field voltage $v_{F}$.

\section{CONTROL DESIGN}

In this Section the Sliding Mode Control technique is applied to an isolated wound rotor synchronous generator. First, after defining the sliding surface, the equivalent control and the equilibria points are computed. Then, an Ideal Sliding Dynamics study validates the selected sliding surface and, finally, the controller is obtained.

\section{A. Sliding surface and equivalent control}

According to the control goals we define sliding surface $s(x)$ as

$$
s(x)=V_{s}^{2}-V_{r e f}^{2} .
$$

Note that, we take $V_{s}^{2}$ to avoid the square root function of (4). Using (4) and (2) we can express the sliding surface with the state variables

$$
s(x)=R_{L}^{2}\left(i_{d}^{2}+i_{q}^{2}\right)-V_{r e f}^{2} .
$$

The equivalent control is defined so that $\dot{s}=0$,

$$
\frac{\partial s}{\partial x} \frac{\mathrm{d} x}{\mathrm{~d} t}=0 .
$$

Then, from (3), (7) and (1),

$$
\frac{\partial s}{\partial x} L^{-1}\left(A x+G u_{e q}\right)=0 .
$$

Hence

$$
u_{e q}=-\left(\frac{\partial s}{\partial x} L^{-1} G\right)^{-1} \frac{\partial s}{\partial x} L^{-1} A x,
$$

or, replacing the matrices and the partial derivatives,

$$
\begin{aligned}
u_{e q}= & R_{F} i_{F}-\frac{L_{F}}{L_{m}}\left(R_{s}+R_{L}\right) i_{d}+\omega L_{m} i_{q} \\
& -\frac{\mu}{L_{s}}\left(\frac{\left(R_{s}+R_{L}\right)}{L_{m}} i_{q}+\omega i_{F}\right) \frac{i_{q}}{i_{d}}
\end{aligned}
$$




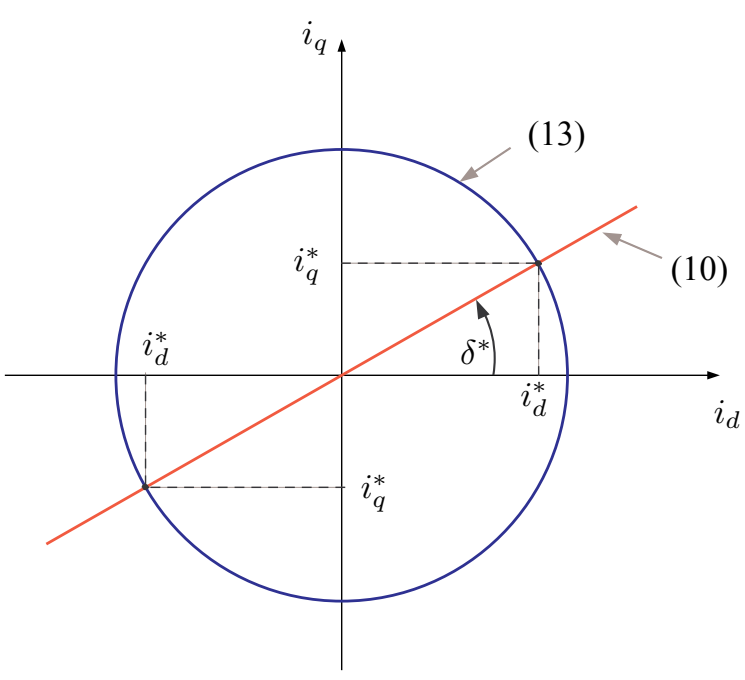

Fig. 3. Equilibrium point distribution.

where $\mu=L_{s} L_{F}-L_{m}^{2}$, which is positive.

The sliding surface is a cylinder in the three-dimensional space $\left(i_{d}, i_{q}, i_{F}\right)$. Sliding motion can be expected only in the cylinder subspace defined by $i_{d} \neq 0$ where transversallity condition holds. Actually, in order to the equivalent control be bounded, a closed set must be taken.

\section{B. Equilibrium points}

Let us to compute the equilibrium points. From (3) and imposing (5) the equilibrium point must satisfy

$$
\begin{aligned}
0 & =-\left(R_{s}+R_{L}\right) i_{d}^{*}+\omega L_{s} i_{q}^{*} \\
0 & =-\omega L_{s} i_{d}^{*}-\left(R_{s}+R_{L}\right) i_{q}^{*}-\omega L_{m} i_{F}^{*} \\
0 & =-R_{F} i_{F}^{*}+v_{F}^{*} \\
V_{\text {ref }}^{2} & =R_{L}^{2}\left(i_{d}^{* 2}+i_{q}^{* 2}\right) .
\end{aligned}
$$

The $i_{d}^{*}$ and $i_{q}^{*}$ values can be computed as follows. Note that (10) and (13) can be represented as the intersection of a circle with a straight line, see Fig. 3.

Defining $\delta^{*}$ such that

$$
\begin{aligned}
i_{d}^{*} & =\frac{V_{r e f}}{R_{L}} \cos \delta^{*} \\
i_{q}^{*} & =\frac{V_{r e f}}{R_{L}} \sin \delta^{*}
\end{aligned}
$$

equation (13) is automatically achieved. Then, replacing (14) and (15) in (10) yields

$$
-\frac{R_{s}+R_{L}}{R_{L}} V_{\text {ref }} \cos \delta^{*}+\frac{\omega L_{s}}{R_{L}} V_{\text {ref }} \sin \delta^{*}=0
$$

which allows to obtain the $\delta^{*}$ value

$$
\delta^{*}=\arctan \left(\frac{R_{s}+R_{L}}{\omega L_{s}}\right) .
$$

From (11) and (13) the field current equilibrium value is

$$
i_{F}^{*}=-\frac{V_{r e f}}{\omega L_{m} R_{L}}\left(\omega L_{s} \cos \delta^{*}+\left(R_{s}+R_{L}\right) \sin \delta^{*}\right),
$$

and, finally, using (12) the control value at the equilibrium is easily obtained,

$$
v_{F}^{*}=-\frac{R_{F}}{\omega L_{m} R_{L}} V_{\text {ref }}\left(\omega L_{s} \cos \delta^{*}+\left(R_{s}+R_{L}\right) \sin \delta^{*}\right)
$$

Note that $v_{F}^{*}=u_{e q}\left(i_{d}^{*}, i_{q}^{*}, i_{F}^{*}\right)$.

\section{Ideal Sliding Dynamics}

The ISD is defined on the sliding surface presumed that it is flow invariant. Since a resistive load is presumed, in Equation (6) it has been written as,

$$
0=R_{L}^{2}\left(i_{d}^{2}+i_{q}^{2}\right)-V_{r e f}^{2},
$$

hence,

$$
i_{d}= \pm \sqrt{\frac{V_{r e f}^{2}}{R_{L}^{2}}-i_{q}^{2}}
$$

This dynamics is defined on the subset of the cylinder where the transversallity condition holds. This subset has two connected components that correspond to the sign of (18). Each component contains an equilibria for Equations (14), (15) and (17). From now on, the positive square root sign is analyzed. The ISD is obtained from the remaining dynamics of $i_{d}$ and $i_{F}$ when (18) is satisfied. From the third row of (3), with the equivalent control (9) and tacking into account the positive sign of (18), the $i_{F}$ dynamics can be written as

$$
\left(\sqrt{\frac{V_{r e f}^{2}}{R_{L}^{2}}-i_{q}^{2}}\right) \frac{\mathrm{d} i_{F}}{\mathrm{~d} t}=-\frac{R_{s}+R_{L}}{L_{m}} \frac{V_{r e f}^{2}}{R_{L}^{2}}-\omega i_{q} i_{F} .
$$

Similarly, $i_{q}$ dynamics is obtained from first row of (3). Summarizing, the Ideal Sliding Dynamics is given by Equation (6) and ,

$$
\begin{aligned}
\frac{\mathrm{d} i_{q}}{\mathrm{~d} t} & =-\omega \sqrt{\frac{V_{r e f}^{2}}{R_{L}^{2}}-i_{q}^{2}}-\frac{R_{s}+R_{L}}{L_{s}} i_{q}-\omega \frac{L_{m}}{L_{s}} i_{F}(19) \\
\frac{\mathrm{d} i_{F}}{\mathrm{~d} t} & =-\frac{1}{\sqrt{\frac{V_{r e f}^{2}}{R_{L}^{2}}-i_{q}^{2}}}\left(\frac{R_{s}+R_{L}}{L_{m}} \frac{V_{r e f}^{2}}{R_{L}^{2}}+\omega i_{q} i_{F}\right)(20)
\end{aligned}
$$

In order to rewrite the ISD, let us define $a=\frac{R_{s}+R_{L}}{L_{s}}$, $c=\frac{R_{s}+R_{L}}{L_{m}}$ and $Y^{2}=\frac{V_{r e f}^{2}}{R_{L}^{2}}$. Note that $a, c, Y>0$. Equations (19) and (20) simplifies in

$$
\begin{aligned}
\frac{\mathrm{d} i_{q}}{\mathrm{~d} t} & =-\omega \sqrt{Y^{2}-i_{q}^{2}}-a i_{q}-\omega \frac{a}{c} i_{F}, \\
\frac{\mathrm{d} i_{F}}{\mathrm{~d} t} & =-\frac{1}{\sqrt{Y^{2}-i_{q}^{2}}}\left(c Y^{2}+\omega i_{q} i_{F}\right) .
\end{aligned}
$$

Since this system is highly non-linear, the stability of the small-signal model around the equilibrium point will be analysed. The small-signal model is a second order linear system. Hence it is stable if, and only if, all the coefficients of the characteristic polynomial have the same sign. The 
Jacobian of the ISD defined by Equations (21) and (22), evaluated at the equilibria, yields

$$
J a c_{I S D}=\left(\begin{array}{cc}
0 & -\omega \frac{a}{c} \\
\omega \frac{c}{a} \frac{1}{\cos ^{2} \delta^{*}} & -\omega \tan \delta^{*}
\end{array}\right)
$$

and the characteristic polynomial,

$$
\lambda^{2}-\operatorname{trace}\left(J a c_{I S D}\right) \lambda+\left|J a c_{I S D}\right| .
$$

At this point, two conditions are necessary and sufficient to assure stability around the equilibrium point. On the one hand, the determinant of $J a c_{I S D}$ must be positive,

$$
\operatorname{det}\left(J a c_{I S D}\right)=\frac{1}{\cos ^{2} \delta^{*}} \omega^{2}>0
$$

which is clearly achieved. On the other hand, the trace of $J a c_{I S D}$ must be negative. From (23)

$$
\operatorname{tr}\left(\operatorname{Jac}_{I S D}\right)=-\omega \tan \delta^{*} .
$$

Finally, using (16), it is clear that this second condition is also achieved,

$$
\operatorname{tr}\left(J a c_{I S D}\right)=-\frac{R s+R_{L}}{L_{s}} .
$$

The small-signal analysis for the equilibrium point in the connected component corresponding to the negative sign of (18) also yields a stable equilibrium point.

Numerical analysis can also be done. Figure 4 shows the vector field and a trajectory of the dynamical system defined by (19) and (20), for a given initial condition, $i_{q}(0)=0$ and $i_{F}(0)=0$. The parameters values are the same than the used in Section IV, where numerical simulations are performed. The equilibrium point, computed from (15) and (17) is $i_{q}^{*}=$ $51.138 \mathrm{~A}$ and $i_{F}^{*}=-214.72 \mathrm{~A}$.

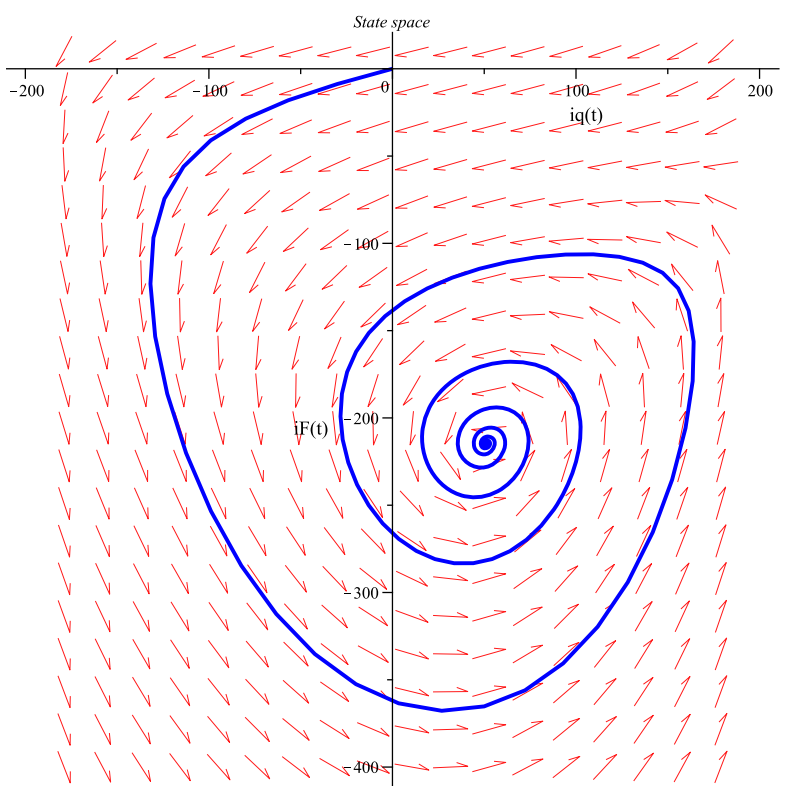

Fig. 4. State space: vector field and trajectory for a given initial condition, $i_{q}(0)=0$ and $i_{F}(0)=0$.
Notice that the trajectories around the equilibrium point are stable, but far to it they tend to the $-\frac{V_{r e f}}{R_{L}}$ boundary of the dynamics domain defined by $i_{q}=\left(-\frac{V_{r e f}}{R_{L}}, \frac{V_{r e f}}{R_{L}}\right)$. In this sense, to ensure the expected behaviour, an exhaustive analysis of the complete nonlinear dynamics should be done.

\section{Sliding Mode Controller}

The Sliding Mode Controller must to ensure that the system goes to the sliding surface and when it occurs keeps on this. In our case, the desired sliding surface was defined in (5).

Consider the following Lyapunov function

$$
V=\frac{1}{2} s^{2},
$$

which is positive and bounded from below. To stabilize the closed loop system the derivative of (24) must be negative, $\dot{V}<0$ which, after some algebra, is equivalent to,

$$
s \frac{\partial s}{\partial x} L^{-1}(A x+G u)<0 .
$$

Last equation can be also written as

$$
s \frac{\partial s}{\partial x} L^{-1}\left(A x+G u-G u_{e q}+G u_{e q}\right)<0,
$$

and tacking into account (8),

$$
s \frac{\partial s}{\partial x} L^{-1} G\left(u-u_{e q}\right)<0 .
$$

Finally, from (25), the control action defined by

$$
u=u_{e q}-k \operatorname{sign}\left(s \frac{\partial s}{\partial x} L^{-1} G\right),
$$

fulfils the stability condition, $\dot{V}<0$,

$$
\dot{V}=-k\left|s \frac{\partial s}{\partial x} L^{-1} G\right| \leq 0 .
$$

Evaluating $\left(s \frac{\partial s}{\partial x} L^{-1} G\right)$ in (26), the control law yields

$$
u=u_{e q}-k \operatorname{sign}\left(-s \frac{2 R_{L}^{2} L_{m}}{L_{s} L_{F}-L_{m}^{2}} i_{d}\right),
$$

and tacking into account that $\frac{2 R_{L}^{2} L_{m}}{L_{s} L_{F}-L_{m}^{2}}>0$

$$
u=u_{e q}-k \operatorname{sign}\left(-s i_{d}\right) .
$$

\section{E. Implementable control law}

In this system, the control action $v_{F}$ is usually implemented using a DC-DC power converter which commutes between two discrete signal values, $-V_{D C}$ and $V_{D C}$. Thus, the control law proposed in the previous section can be modified as follows.

Consider that the switching voltage control $u \in\left\{u_{1}, u_{2}\right\}$, and that the equivalent control lies in the interval $\left(u_{1}, u_{2}\right)$. From the stability condition obtained in (25), the control law can be defined by

$$
u=\left\{\begin{array}{lll}
u^{-} & \text {if } & s \frac{\partial s}{\partial x} L^{-1} G>0 \\
u^{+} & \text {if } & s \frac{\partial s}{\partial x} L^{-1} G \leq 0
\end{array},\right.
$$


which also assures that $\dot{V} \leq 0$.

Particularising these results to our case and tacking into account that $\frac{2 R_{L}^{2} L_{m}}{L_{s} L_{F}-L_{m}^{2}}>0$, the control action, $v_{F}$, is defined by

$$
v_{F}=\left\{\begin{array}{ll}
-V_{D C} & \text { if } \quad-s i_{d}>0 \\
V_{D C} & \text { if } \quad-s i_{d} \leq 0
\end{array} .\right.
$$

\section{SIMULATIONS}

In this section, a set of simulations using the designed controller are presented. The WRSM has the following parameters: $L_{s}=0.0262487 \mathrm{H}, R_{s}=0.181 \Omega, L_{m}=0.02529 \mathrm{H}$, $L_{F}=0.027185 \mathrm{H}, R_{F}=0.1002 \mathrm{H}$. The mechanical speed is fixed at $\omega=314 \mathrm{rad} \mathrm{s}^{-1}$ and the initial value of the load resistance is $R_{L}=2 \Omega$. The desired stator voltage amplitude, $V_{s}$, is set in $V_{\text {ref }}=400 \mathrm{~V}$.

Substituting machine parameters and the expected load in (9), we determine $u_{e q}$ at the desired operation point. Namely, $u_{e q}^{*}= \pm 21.51 \mathrm{~V}$. From this, the field DC voltage is set to $V_{D C}= \pm 40 \mathrm{~V}$

In order to implement the discrete time controller a hysteresis block is added to the (27) condition. The hysteresis parameters are set depending on the maximum chattering value and the minimum switching time of the DC-DC power converter. In the numerical experiment the load resistance varies from $R_{L}=2 \Omega$ to $R_{L}=1.9 \Omega$ at $t=1 \mathrm{~ms}$.

Figure 5 shows that the voltage amplitude is regulated. Even if the $R_{L}$ value is suddenly modified, the steady state value of the output is the desired one, and also the convergence-time is small.

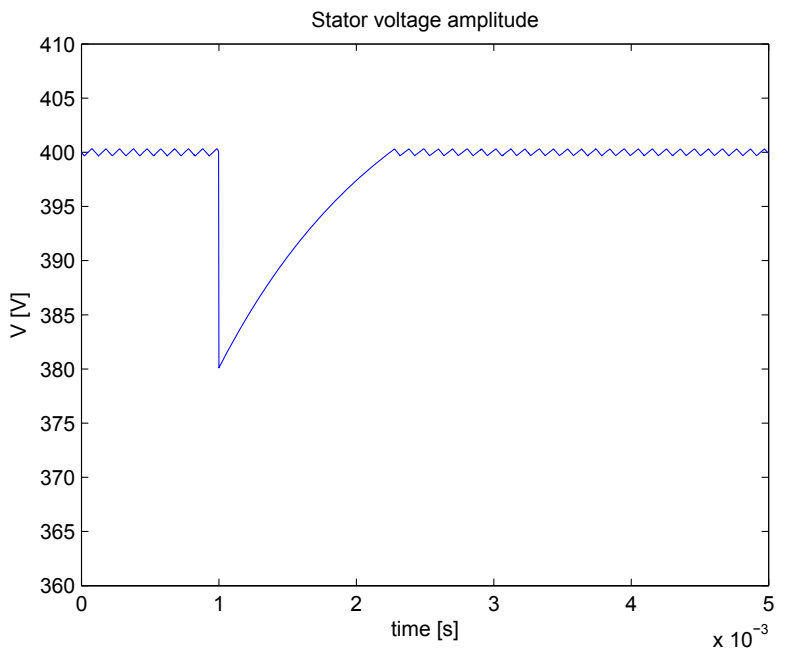

Fig. 5. Simulation results: stator voltage amplitude, $V$.

Figures 6 and 7 show the field voltage, $v_{F}$, which acts as a control action, and the sliding surface $s$, defined in (6). Note that $v_{F}$ commutes between $\pm V_{D C}$, and that the sliding surface oscillates around zero.

Finally, in Fig. 8, the equivalent control is also depicted to show that it remains in the expected boundary, see previous Section.

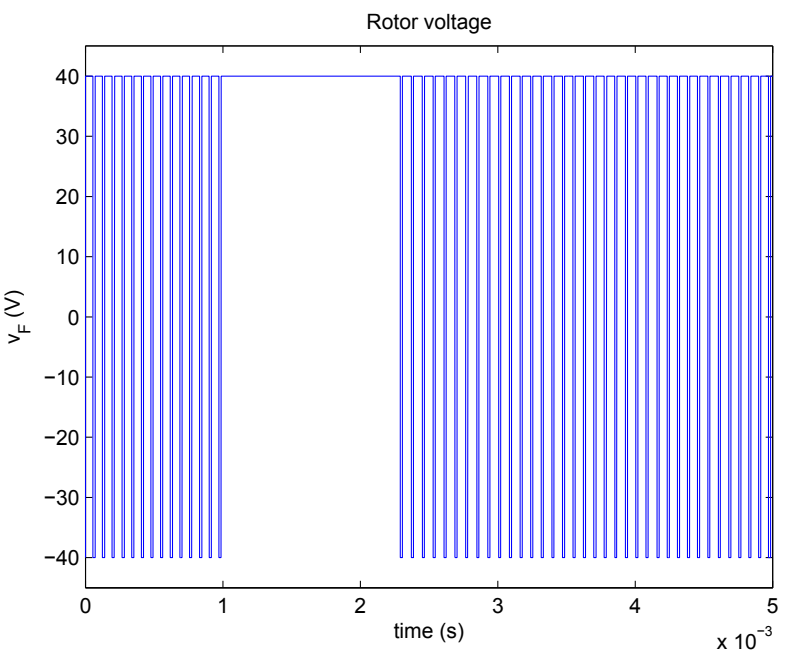

Fig. 6. Simulation results: field voltage control action, $v_{F}$.

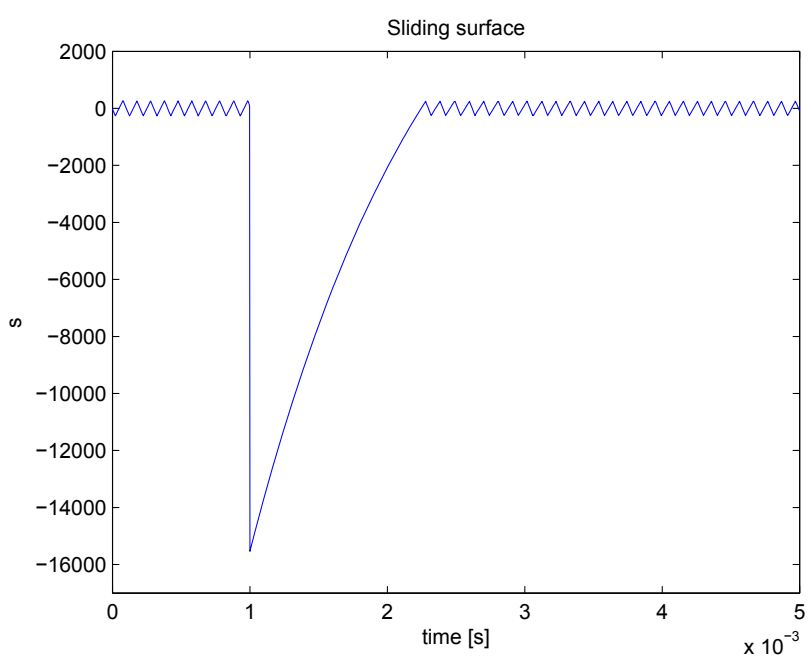

Fig. 7. Simulation results: sliding surface, $s$.

\section{CONCLUSIONS}

In this paper a Sliding Mode controller has been designed for a WRSM acting as a generator for an isolated resistive load. The obtained control law regulates the stator voltage amplitude irrespectively of the load value. The stability of the closed loop system has been discussed through the smallsignal model around the equilibrium point.

The next step is to fully study the nonlinear ISD.

Experimental validation with a real plant using the presented control law will be considered. Future research also includes synthesis and analysis problems for the case of nonlinear and dynamical loads.

\section{ACKNOWLEDGMENTS}

A. Dòria-Cerezo and E. Fossas were partially supported by the Spanish government research project DPI2007-62582. 


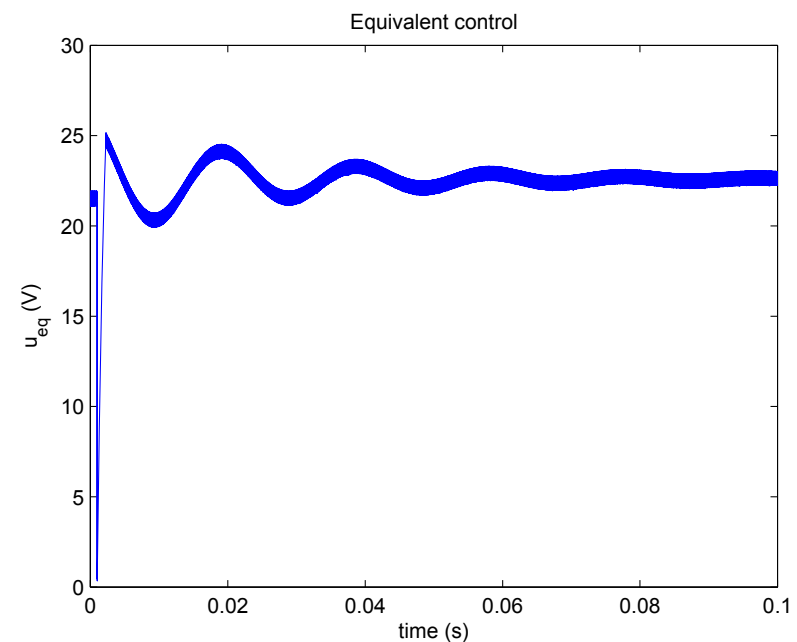

Fig. 8. Simulation results: equivalent control, $u_{e q}$.

\section{REFERENCES}

[1] C. Rossi, D. Casadei, A. Pilati, and M. Marano. Wound rotor salient pole synchronous machine drive for electric traction. In Proc. IEEE Industry Applications Conference, 2006.

[2] R.C. Schaefer. Excitation control of the synchronous motor. IEEE Trans. on Industry Applications, 35(3):694-702, 1999.

[3] W. Leonhard. Control of electric drives. Springer, 1995.

[4] M.K. Senesky, P. Tsao, and S.R. Sanders. Simplified modelling and control of a synchronous machine with variable-speed six-step drive. In Proc. IEEE Applied Power Electronics Conference and Exposition, 2004.

[5] E. Ho and P.C. Sen. High-performance decoupling control techniques for various rotating field machines. IEEE Trans. on Industrial Electronics, 42(1):40-49, 1995.

[6] C. Batlle, A. Dòria-Cerezo, and G. Espinosa. Simultaneous idapassivity-based control of a wound rotor synchronous motor. In Proc. IEEE Conf. on Decision and Control, 2008.

[7] J. Cabrera-Vázquez, A.G. Loukianov, J.M. Cañedo, and V.I. Utkin. Robust controller for synchronous generator with local load via vsc. Electrical Power and Energy Systems, 29:348-359, 2007.

[8] D. Biel and E. Fossas. Smc application in power electronics. In A. Sabanovic, L.M. Fridman, and S. Spurgeon, editors, Variable Structure Systems: from Principles to Implementation, volume 66, pages 265-284, London, 2004. IEE Control Series.

[9] V. Utkin, J. Guldner, and J. Shi. Sliding Mode Control in Electromechanical Systems. Taylor and Francis, 1999.

[10] C. Namuduri and P.C. Sen. A servo-control system using a selfcontrolled synchronous motor (scsm) with sliding mode controller. IEEE Trans. on Industry Applications, 23(2):283-295, 1987.

[11] F. Valenciaga and P.F. Puleston. High-order sliding control for a wind energy conversion system based on a permanent magnet synchronous generator. IEEE Trans. on Energy Conversion, 23(3), 2008. 